\title{
Exacerbations of Chronic Obstructive Pulmonary Disease Tool to assess the efficacy of acute treatment
}

This article was published in the following Dove Medical Press journal: International Journal of COPD

Hye Sook Choi,' Yong Bum Park, ${ }^{2,3}$ Kyeong Cheol Shin, ${ }^{4}$ Seung Hun Jang, ${ }^{3,5}$ Kang Hyeon Choe, ${ }^{6}$ Young Sam Kim, ${ }^{7}$ Sun Young Kyung, ${ }^{8}$ Sung Ho Yoon, ${ }^{9}$ Ju Sang Kim, ${ }^{10}$ Sung Soo Jung ${ }^{11}$

'Division of Pulmonary, Allergy and Critical Care Medicine, Department of Internal Medicine, Kyung Hee University Medical Center, Seoul, Republic of Korea; ${ }^{2}$ Division of Pulmonary, Allergy, and Critical Care Medicine, Department of Medicine, Hallym University Sacred Heart Hospital-Kangdong, Seoul, Republic of Korea; ' $D$ epartment of Internal Medicine, Hallym University College of Medicine, Chuncheon, Republic of Korea; ${ }^{4}$ Division of Pulmonology and Allergy, Department of Interna Medicine, Yeungnam University Hospital, Daegu, Republic of Korea; ${ }^{5}$ Division of Pulmonary, Allergy, and Critical Care Medicine, Department of Medicine, Hallym University Sacred Heart Hospital, Hallym University College of Medicine, Anyang, Republic of Korea; ${ }^{6}$ Division of Pulmonary, Allergy and Critical Care Medicine, Department of Internal Medicine, Chungbuk National University Hospital, Cheongju, Republic of Korea; ${ }^{7}$ Division of Pulmonary, Allergy and Critical Care Medicine, Department of Internal Medicine, Yonsei University Health System, Seoul, Republic of Korea; ${ }^{8}$ Division of Pulmonary, Allergy and Critical Care Medicine, Department of Internal Medicine, Gachon

University Gil Medical Center, Incheon, Republic of Korea; ' ${ }^{\circ}$ ivision of Pulmonary, Allergy and Critical Care Medicine, Department of Internal Medicine. Chosun University Hospital, Gwangju, Republic of Korea; ${ }^{10}$ Division of Pulmonary, Allergy and Critical Care Medicine, Department of Internal Medicine, The Catholic University, Incheon St Mary's Hospital, Incheon, Republic of Korea; "Department of Internal Medicine, Division of Pulmonary and Critical Care Medicine, Chungnam National University College of Medicine, Daejeon, Republic of Korea
Correspondence: Sung Soo Jung

Division of Pulmonary and Critical Care Medicine

Chungnam National University School of Medicine, 282

Munhwa-ro, Jung-gu Daejeon 35015, Republic of Korea

Tel +82422808103

Fax +82 422575753

Email sungsoojung09@cnu.ac.kr
Background and objective: The Exacerbations of Chronic Obstructive Pulmonary Disease Tool-Patient-Reported Outcomes (EXACT-PRO) has been suggested as a reliable and valid measure for early assessment of COPD exacerbations and perceived recovery. However, there has been no evidence for EXACT-PRO efficacy in assessing recovery from treatment in a randomized controlled trial. The study evaluated the reliability, validity, and responsiveness of EXACT-PRO for the evaluation of the efficacy of acute treatment in patients with COPD exacerbation.

Methods: In a Phase III randomized controlled study for assessing the efficacy of antibiotic treatment on COPD exacerbation, EXACT-PRO was evaluated in the responders and non-responders. Results: A total of 295 patients were analyzed (259 responders and 37 non-responders). Cronbach's $\alpha$ was 0.96 for EXACT total, 0.96 for the breathlessness domain, 0.89 for the cough and sputum domain, and 0.93 for the chest symptoms domain. The EXACT score correlated with the COPD assessment test (CAT) score $(r=0.8, P<0.01)$. A stronger decrease in the EXACT score was found in the responder group than in the non-responder group from the fifth day after treatment. The difference in the EXACT score from exacerbation onset to recovery was -6.3 in responders and -1.9 in non-responders $(P=0.01)$.

Conclusion: EXACT-PRO is a comprehensive and sensitive method for assessing symptomatic resolution of COPD exacerbations during treatment.

Keywords: The Exacerbations of Chronic Obstructive Pulmonary Disease Tool, EXACT, Patient-Reported Outcomes, PRO, COPD, exacerbation

\section{Plain language summary}

We assessed the usefulness of the Exacerbations of Chronic Obstructive Pulmonary Disease Tool-Patient-Reported Outcomes (EXACT-PRO) during exacerbation and recovery period of COPD. EXACT-PRO score well correlated with treatment outcome. A stronger decrease in the EXACT score was found in the responder group after treatment of COPD exacerbation. EXACT-PRO is a useful tool for assessing COPD exacerbations and recovery.

\section{Introduction}

COPD exacerbations are defined as an acute worsening of respiratory symptoms beyond normal day-to-day variability, which need additional therapy and carry the risk of adverse health events that may affect the patient at some point in the future. ${ }^{1}$ Many studies have tried to detect exacerbations at the initial phase using clinical or laboratory parameters. Nonetheless, many exacerbation cases are not reported to health care professionals, ${ }^{2}$ and thus, COPD patients need to receive education 
about exacerbation symptoms and when to visit a health care facility. ${ }^{1}$ Standardizing the assessment of COPD exacerbation symptoms using a common tool and metric will promote understanding exacerbations, including the prodromal, acute, and recovery phases, and the effects of treatment. ${ }^{3}$

The EXACT-PRO instrument designed to directly measure patient-reported symptoms of COPD exacerbation and to standardize the assessment of the patient's condition in order to capture this dynamic process of exacerbations. ${ }^{3,4}$ EXACT is a tool for assessing the frequency, severity, and duration of exacerbations of COPD; $;^{3-5}$ it is also used in clinical trials to test the efficacy of therapies aimed at alleviating or preventing COPD exacerbations ${ }^{6}$ or treating exacerbations. ${ }^{3,7}$ However, to date, there are no data to evaluate the performance of EXACT in a randomized controlled clinical trial that would assess the efficacy of treatment in patients with COPD exacerbation at baseline. ${ }^{3}$

Elevated white blood cell count and its subtypes are systemic inflammatory markers. The neutrophil-to-lymphocyte ratio (NLR), which calculated from complete blood cell count with differential, is a rapid, inexpensive, and widely available marker of inflammation in clinical practice. We hypothesized that elevated NLR in COPD exacerbations decreases in the recovery phase following treatment.

One aim of the present study was to evaluate the performance of EXACT in the assessment of the efficacy of acute treatment. Second, we planned to validate the Korean version of EXACT-PRO in Korean COPD patients with exacerbations as a sensitive tool to assess recovery from exacerbation. Third, we evaluate NLR changes during recovery according to the treatment response.

\section{Methods}

\section{Study design, sample, and procedures}

A prospective, multicenter, double-blind, double-dummy, randomized, controlled, parallel-group, Phase III noninferiority clinical trial designed to compare oral zabofloxacin with moxifloxacin for the treatment of patients with COPD exacerbations was performed between September 2012 and February 2014 in Korea. The study is registered with ClinicalTrials.gov (registration number NCT01658020) and Clinical Research Information Service (KCT0000532). The study was approved by independent ethics committees (CNUH 2012-07-013) at each center before study initiation. All patients provided written informed consent. Study design and results on primary outcomes about this study were published in a paper. ${ }^{8}$ A total of 345 COPD patients with exacerbations were enrolled, and all of them received one of the study drugs for seven consecutive days.

\section{Measures}

\section{Patient-reported measures}

The Korean version of the EXACT-PRO questionnaire was produced from the original English version by following a standard translation and back-translation process. The participating patients recorded the Korean version of the EXACT pen diary booklet on days $1-7$ and 36 . The participants were instructed to complete the pen diary each evening before bedtime.

EXACT consists of 14 items in three domains as follows: Breathlessness, Cough \& Sputum, and Chest Symptoms. The EXACT total score is computed across the 14 items and has an interval-level scale ranging from 0 to 100 , with higher scores indicating a more severe condition. The score of each domain also ranges from 0 to 100, with higher scores indicating more severe symptom. ${ }^{3}$

The COPD assessment test (CAT) questionnaire was administered on days $1-7$ and 36 according to study design.

\section{Laboratory measures}

Blood test was done on day 1 (visit 1, V1), days $10 \pm 3$ (visit 3, V3), and days 36 7 (visit 4, V4) and included complete blood cell count with differential count. We calculated the NLR for each day.

\section{Clinical assessment}

The patients who improved in terms of dyspnea or sputum purulence or volume on V3 after acute treatment were defined as "responders", and those who did not were defined as "non-responders". 8

\section{Statistical analyses}

All statistical analyses were performed using SPSS version 24 (IBM Corporation, Armonk, NY, USA). To compare baseline characteristics between responders and non-responders, a paired $t$-test (continuous variables) and a chi-squared test were used. To compare the changes in the EXACT score, the CAT score, and the NLR on the treatment each day between responders and non-responders, a paired $t$-test was used. In the main text, the data are presented as mean $\pm \mathrm{SD}$ or percentage. In the figures, the data are presented as mean \pm standard error of mean. To test the reliability of the Korean version of EXACT questionnaire items, internal consistency was assessed using Cronbach's $\alpha$. To test validity, the relationship 
between EXACT total score and CAT score was assessed using Pearson's correlation coefficient. The $P$-values of $<0.05$ were considered statistically significant.

\section{Results}

\section{Patients' characteristics}

In total, 322 patients completed the EXACT pen diary on consecutive days 1-7; among them, 295 patients could be evaluated for the clinical response to the treatment. In total, 287 patients also completed the diary on day 36 , and all of them could be evaluated for the clinical response to the treatment. Clinical characteristics at baseline are shown in Table 1. Mean post-bronchodilator $\mathrm{FEV}_{1}$ was $49.5 \%$ of predicted value. Mean EXACT total score on day 1 was 46.1 . There were no differences in EXACT total score or three domain scores between responders and non-responders on day 1 . Mean NLR on day 1 was 3.9, with a trend toward a lower NLR at baseline in the non-responders (NLR $=3.1$, 95\% CI: 2.75-3.45) compared to responders (NLR $=4.1$, 95\% CI: 3.61-4.59). But there are no statistical differences in NLR between responders and non-responders. The CAT score and the presence of chronic bronchitis were similar in both groups.
Patients with chronic bronchitis showed higher EXACT and CAT scores than patients without chronic bronchitis (Table 2).

\section{Reliability of EXACT}

Internal consistency of Cronbach's $\alpha$ for the EXACT total score, breathlessness domain, and chest symptoms domain exceeded 0.9 (Table 3 ). These data suggested that the EXACT questionnaire items had good internal consistency and were reliable.

\section{Validity of EXACT}

Correlations between the EXACT scores and the CAT score are presented in Table 4. The EXACT total score, breathlessness domain score, and chest symptoms domain score were significantly related to the CAT score on day 1 (Pearson correlation coefficient, $r=0.80,0.71,0.61$, respectively; all $P<0.01)$. The EXACT total score and CAT score on days 7 and 36 showed strong relationship $(r=0.79,0.80$, respectively; $P<0.01)$. The cough and sputum domain score and the CAT score showed a moderate relationship on days 1,7 , and 36 .

$\mathrm{FEV}_{1} \%$ predicted showed a weak inverse relationship with the EXACT total score and the CAT score on day 1

Table I Baseline characteristics of patients

\begin{tabular}{|c|c|c|c|c|}
\hline Characteristics & Total $(n=295)$ & Responders $(n=258)$ & Non-responders $(n=37)$ & $P$-value \\
\hline Age, years & $70 \pm 7.6$ & $69.8 \pm 7.5$ & $7 I \pm 7.5$ & 0.41 \\
\hline Sex, male, n (\%) & $272(92)$ & $240(92.7)$ & $32(86.5)$ & 0.18 \\
\hline Zabofloxacin, n (\%) & $152(51.5)$ & $133(5 \mid .6)$ & $19(5 \mathrm{I})$ & 0.98 \\
\hline $\mathrm{BMI}, \mathrm{kg} / \mathrm{mm}^{2}$ & $22.1 \pm 3.3$ & $22.1 \pm 3.3$ & $22.1 \pm 3.6$ & 0.99 \\
\hline Smoking, packs/year & $29.5 \pm 18.9$ & $28.7 \pm 18.6$ & $38.8 \pm 21.4$ & 0.21 \\
\hline Exacerbation history, prior 12 months & $I \pm 0 . I$ & $\mathrm{I} \pm 0 . \mathrm{I}$ & $\mathrm{I} \pm 0.0$ & 0.53 \\
\hline \multicolumn{5}{|l|}{ Spirometry } \\
\hline $\mathrm{FEV}_{1}, \mathrm{~L}$ & $1.4 \pm 0.5$ & $1.4 \pm 0.5$ & $1.3 \pm 0.4$ & 0.31 \\
\hline $\mathrm{FEV}_{1} \%$ predicted & $49.5 \pm 17.7$ & $49.4 \pm 17.6$ & $50.1 \pm 18.4$ & 0.84 \\
\hline \multicolumn{5}{|l|}{ EXACT score, day I } \\
\hline Total score & $46.1 \pm 11.0$ & $46.1 \pm 11.3$ & $46.4 \pm 8.7$ & 0.84 \\
\hline Breathlessness & $50.8 \pm 19.8$ & $50.6 \pm 20.3$ & $52.2 \pm 15.9$ & 0.65 \\
\hline Cough and sputum & $44.4 \pm 16.3$ & $44.8 \pm 16.4$ & $41.7 \pm 15.3$ & 0.27 \\
\hline Chest symptoms & $34.2 \pm 19.7$ & $34.1 \pm 20.0$ & $35.5 \pm 17.6$ & 0.67 \\
\hline CAT score, day I & $23 \pm 7.3$ & $22.9 \pm 7.4$ & $23.7 \pm 6.9$ & 0.54 \\
\hline Chronic bronchitis, n (\%) & $116(39.3)$ & $104(40.3)$ & $12(32.4)$ & 0.47 \\
\hline White blood cell count $/ \mu \mathrm{L}$ & $8,620 \pm 3,168$ & $8,680 \pm 3,263$ & $8,202 \pm 2,410$ & 0.39 \\
\hline Neutrophils, \% & $62.6 \pm 16.7$ & $62.8 \pm 17.2$ & $61.1 \pm 12.7$ & 0.57 \\
\hline Lymphocytes, \% & $22.4 \pm 10.3$ & $22.1 \pm 10.5$ & $24.1 \pm 8.5$ & 0.26 \\
\hline NLR & $3.9 \pm 3.8$ & $4.1 \pm 4.0$ & $3.1 \pm 1.8$ & 0.145 \\
\hline
\end{tabular}

Note: All values are presented as mean \pm SD except where indicated.

Abbreviations: BMI, body mass index; CAT, COPD assessment test; EXACT, Exacerbations of Chronic Pulmonary Disease Tool; NLR, neutrophil/lymphocyte ratio. 
Table 2 Symptom score according to chronic bronchitis in patients with COPD exacerbation

\begin{tabular}{|c|c|c|c|c|}
\hline \multirow{2}{*}{$\begin{array}{l}\text { EXACT or } \\
\text { CAT score }\end{array}$} & \multicolumn{2}{|c|}{ Chronic bronchitis } & \multirow{2}{*}{$\begin{array}{l}\text { Mean } \\
\text { difference } \\
(95 \% \mathrm{Cl})\end{array}$} & \multirow[t]{2}{*}{$P$-value } \\
\hline & $\begin{array}{l}\text { No } \\
(n=179)\end{array}$ & $\begin{array}{l}\text { Yes } \\
(n=|| 5)\end{array}$ & & \\
\hline \multicolumn{5}{|l|}{ EXACT score } \\
\hline Day I & $44.4 \pm 9.9$ & $48.9 \pm 12.1$ & $4.5(3.47-5.53)$ & $<0.01$ \\
\hline Day 7 & $39.0 \pm 10.8$ & $42.4 \pm 11.9$ & $3.4(2.24-4.56)$ & 0.01 \\
\hline \multicolumn{5}{|l|}{ CAT score } \\
\hline Day I & $21.2 \pm 6.8$ & $25.8 \pm 7.3$ & $4.6(4.32-4.88)$ & $<0.01$ \\
\hline Day 7 & $18.0 \pm 7.6$ & $21.8 \pm 7.5$ & $3.8(3.45-4.15)$ & $<0.01$ \\
\hline
\end{tabular}

Note: Data are mean \pm SD.

Abbreviations: CAT, COPD assessment test; EXACT, Exacerbations of Chronic Pulmonary Disease Tool.

( $r=-0.19, r=-0.25$, respectively; both $P<0.01$ ). The breathlessness domain score on day 1 showed the strong inverse relationship with $\mathrm{FEV}_{1} \%$ predicted $(r=-0.26, P<0.01)$ among EXACT domains (Table 5).

\section{Responsiveness of EXACT}

There were significant differences in EXACT scores between responders and non-responders from day 5 after the beginning of the treatment $(41.4 \pm 11.2$ vs $45.4 \pm 10.5 ; P=0.03)$, on day 6 (40.7 \pm 11.4 vs $45.0 \pm 10.2 ; P=0.03)$, and on day 7 (39.7 \pm 11.3 vs $44.5 \pm 11.1 ; P=0.01$; Figure 1 ).

Mean differences in EXACT total scores from day 1 to 7 were -6.3 in responders and -1.9 in non-responders $(P<0.01$; Figure 2A). The breathlessness domain score showed a significantly stronger reduction in responders than in non-responders on day 7 ( -8.6 vs $-2.0 ; P=0.03)$, but the cough and sputum domain $(-13.4$ vs $-8.4 ; P=0.09)$ and the chest symptoms domain $(-6.9$ vs $-2.4 ; P=0.16)$ did not show significant differences between responders and non-responders on day 7 (Figure 2B).

\section{Changes in NLR}

Mean changes in NLR during treatment from V1 to V3 were -0.34 in responders and 1.31 in non-responders

Table 3 Reliability: internal consistency in patients with acute exacerbations

\begin{tabular}{|l|l|}
\hline \multirow{2}{*}{ EXACT score } & Internal consistency $(\mathbf{n = 2 9 5 )}$ \\
\cline { 2 - 2 } & Cronbach's $\boldsymbol{\alpha}$ \\
\hline Total score & 0.96 \\
\hline Breathlessness & 0.96 \\
\hline Cough and sputum & 0.89 \\
\hline Chest symptoms & 0.93 \\
\hline
\end{tabular}

Abbreviation: EXACT, Exacerbations of Chronic Pulmonary Disease Tool.
Table 4 Validity: correlation between EXACT scores and CAT scores on days I, 7, and 36

\begin{tabular}{|l|l|l|l|}
\hline \multirow{2}{*}{ EXACT score } & \multicolumn{3}{|l|}{ Coefficients } \\
\cline { 2 - 4 } & Day I & Day 7 & Day 36 \\
\hline Total score & 0.80 & 0.79 & 0.80 \\
\hline Breathlessness & 0.71 & 0.71 & 0.70 \\
\hline Cough and sputum & 0.50 & 0.47 & 0.49 \\
\hline Chest symptoms & 0.61 & 0.64 & 0.68 \\
\hline
\end{tabular}

Notes: Pearson's correlation coefficients between EXACT scores and CAT scores were determined on day I, day 7 , and day 36 . All $P$-values $<0.0$ I.

Abbreviations: CAT, COPD assessment test; EXACT, Exacerbations of Chronic Pulmonary Disease Tool.

$(P<0.01$; Figure 3$)$. NLR values were significantly decreased from V1 to V3 after antibiotics treatment in responders but not in non-responders.

The NLR showed weak relationship with EXACT total score $(r=0.16 ; P<0.01)$ and CAT score $(r=0.16 ; P<0.01)$ on day 1 . There was a weak inverse relationship between the NLR and $\mathrm{FEV}_{1} \%$ predicted $(r=-0.19 ; P<0.01$; Table 5$)$.

\section{Discussion}

Despite the efforts to prevent or at least reduce COPD exacerbations, there has been no standardized, reliable, and valid method for quantifying exacerbations. Clinical studies have used two different definitions of COPD exacerbations as follows: event-based and symptom-based. Event-based exacerbation frequency is defined on the basis of health care use with systemic steroid or antibiotics treatment; ${ }^{9}$ this definition has been used most frequently in clinical studies. However, event-based definition fails to account for unreported events and to standardize the change in symptoms from normal day-to-day variability. ${ }^{2}$ The symptom-based definition assesses this change using a patient-completed symptom diary card. ${ }^{9}$

Initial symptoms and patient-reported signs of exacerbation should be captured by a well-defined and reliable PRO instrument. Exacerbations that are not reported when using

Table 5 Correlations between the EXACT score, CAT score, $\mathrm{NLR}$, and $\mathrm{FEV}, \%$ predicted on day I

\begin{tabular}{|l|l|l|}
\hline \multirow{2}{*}{} & \multicolumn{2}{|l|}{ Coefficients $(\mathbf{9 5} \% \mathbf{C I})$} \\
\cline { 2 - 3 } & FEV $\%$ predicted & NLR \\
\hline EXACT total score & $-0.19^{\mathrm{a}}$ & $0.16^{\mathrm{c}}$ \\
\hline CAT total score & $-0.25^{\mathrm{b}}$ & $0.16^{\mathrm{d}}$ \\
\hline
\end{tabular}

Notes: Pearson's correlation coefficients between: ${ }^{2}$ EXACT scores and FEV $\%$ predicted; ' $\mathrm{CAT}$ scores and $\mathrm{FEV} \%$ predicted; 'EXACT scores and NLR; and ${ }^{\mathrm{d}}$ CAT scores and NLR. All P-values $<0.05$.

Abbreviations: CAT, COPD assessment test; EXACT, Exacerbations of Chronic Pulmonary Disease Tool; NLR, neutrophil/lymphocyte ratio. 


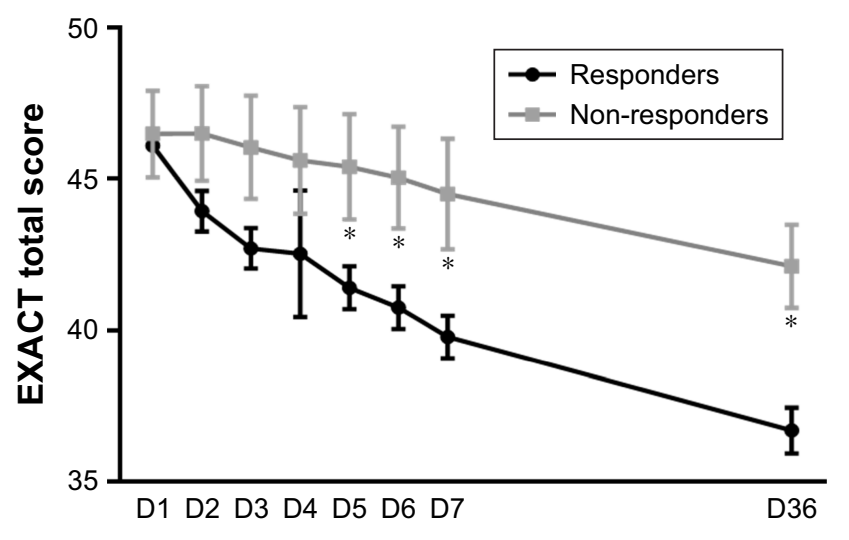

Figure I Serial changes in EXACT total scores of responders and non-responders during acute treatment of COPD exacerbations.

Notes: From day 5 of treatment, EXACT total score was significantly different between the two groups. $* P<0.05$.

Abbreviations: D, day; EXACT, Exacerbations of Chronic Pulmonary Disease Tool.

the event-based definition are expected to be reported when using the symptom-based definition, to be perceived earlier, and to provide an objective outcome measure for frequency, severity, and duration of and recovery from exacerbation. PRO needs to detect and quantify COPD exacerbations. The EXACT-PRO tool was developed to standardize the symptomatic assessment of exacerbations by including unreported events. ${ }^{4}$ The original English version of EXACT-PRO is a self-administered daily e-diary to be completed by respondents each evening just before the bedtime while reflecting on their symptoms today. Changes in the total score are used to define onset and resolution from an exacerbation event and the magnitude of that event. ${ }^{3}$
Standardized PRO provides an objective outcome measure for frequency, severity, and duration of exacerbations that can be used in clinical trials. ${ }^{3}$ Daily administration of EXACT-PRO is essential for capturing changes in the patient's condition over time, including worsening, improvement, and stabilization. The EXACT tool identified more frequent unreported exacerbations than did event-based identification. It is important because unreported exacerbation events have a similar impact on health status to the reported events. ${ }^{10}$

Previous clinical trials suggest that EXACT-PRO is a standardized, reliable, valid tool for assessing frequency, severity, and duration of exacerbations. ${ }^{5-7}$ It is also sensitive to changes during recovery from exacerbations. ${ }^{7}$ However, all the previous studies have been performed in the Western countries.

This is the first study to use EXACT to assess recovery from exacerbations in a randomized clinical trial that evaluated the effects of treatment on COPD patients enrolled in a state of clinical exacerbation at baseline. Some of the results of this study have been previously reported in the form of an abstract. ${ }^{11}$

In a previous study, the EXACT scores differentiated responders and non-responders on day 7 following exacerbation treatment. ${ }^{7}$ Our study supports the previous result that the EXACT instrument is sensitive to changes during recovery from exacerbations. In our study, the EXACT scores differentiated responders and non-responders on the fifth day following treatment: the decreases in the EXACT
A

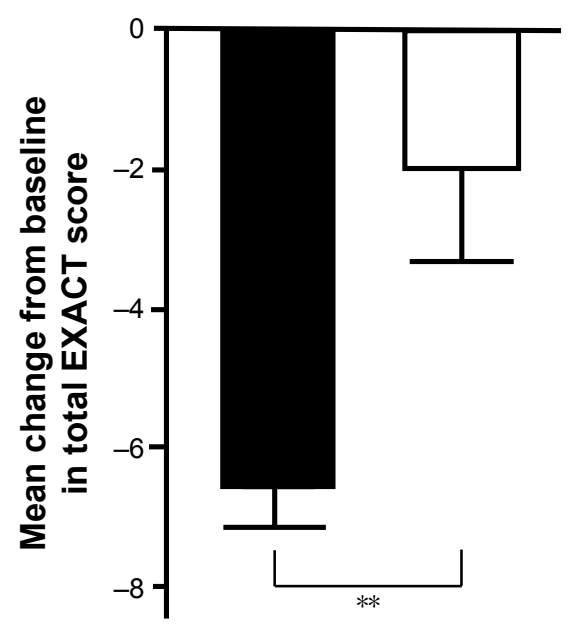

B

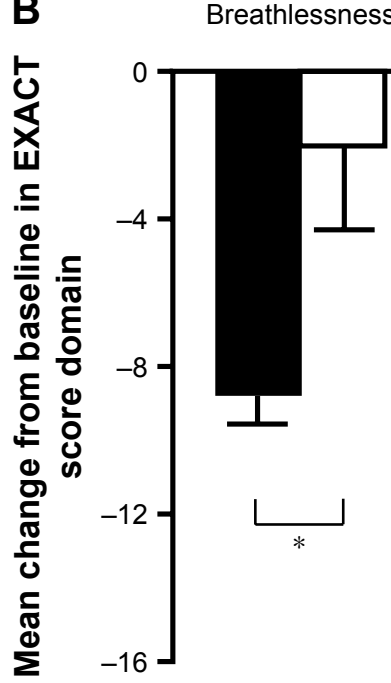

Cough and Chest symptoms Sputum

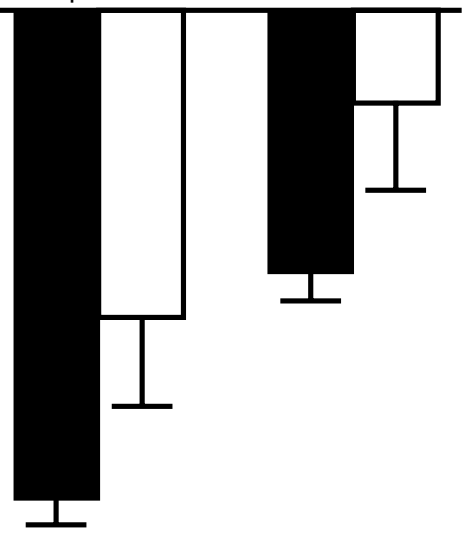

Figure 2 (A) Mean changes in EXACT total scores between days I and 7 showed a significant reduction in responders but not in non-responders. (B) Reduction in the breathlessness score from day I to 7 was significant.

Notes: The reduction in the cough and sputum domain scores and that in the chest symptoms domain score were not significant. $* P<0.05$ and $* * P<0.01$.

Abbreviation: EXACT, Exacerbations of Chronic Pulmonary Disease Tool. 


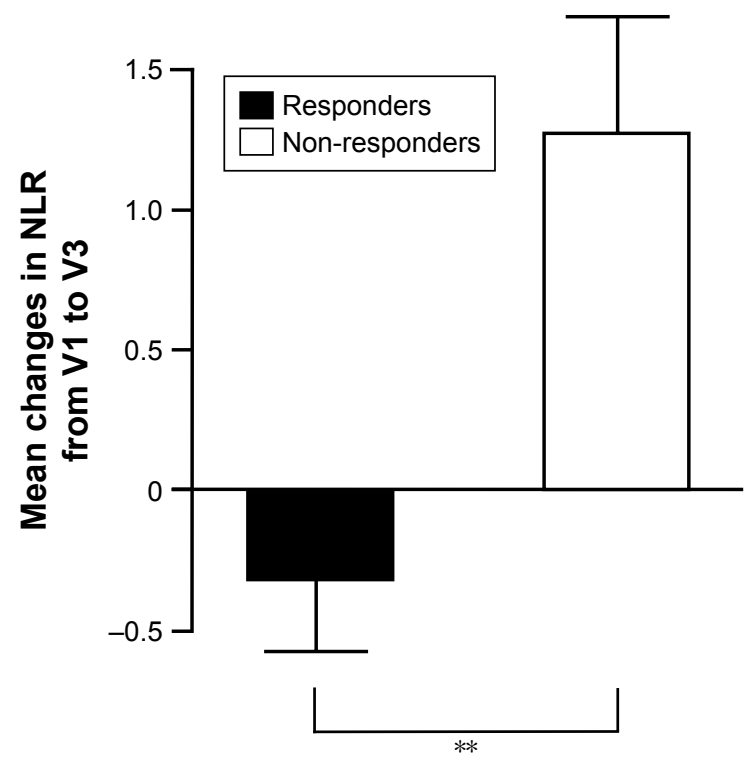

Figure 3 Mean changes in the NLR from VI to V3.

Notes: The NLR decreased significantly in non-responders. $* * P<0.01$. VI, baseline visit; $\mathrm{V} 3$, visit on day $7 \pm 3$.

Abbreviation: NLR, neutrophil/lymphocyte ratio.

total score and especially the decrease in the breathless domain score from exacerbation to recovery were higher in responders than in non-responders. The EXACT is reliable and valid for assessing the severity, frequency, and duration of exacerbations..$^{5-7,12,13}$ This study adds the responsiveness to recovery from exacerbations to the previously reported characteristics of EXACT. Leidy et $\mathrm{al}^{7}$ first reported the sensitivity to improvement in patients with a medically confirmed exacerbation of COPD. Our study is the first report to verify sensitivity to improvement in a Phase III clinical trial. We suggest that the EXACT measure is suitable for clinical trials as a PRO for differentiation of treatment efficacy.

The Cronbach's $\alpha$ value of EXACT total was 0.96 in this study. This is similar to that reported in the original validation study by Leidy et $\mathrm{al}^{7}$ and is higher than the recommended 0.90 threshold. $^{14}$

The CAT is currently recommended for assessing the symptoms and health status of COPD patients ${ }^{1}$ and has shown good measurement properties with good reliability and validity. ${ }^{15}$ The CAT is sensitive to changes in health status ${ }^{16}$ and in systemic inflammation ${ }^{17}$ during COPD exacerbations. The CAT is a useful instrument to assess the efficacy of treatments following COPD exacerbations. ${ }^{17}$ In the first validation study of the EXACT-PRO, the EXACT score was correlated with the St George's Respiratory Questionnaire score $(r=0.64) .^{7}$ In our study, the EXACT scores were significantly correlated with the CAT score. We suggest the usefulness of EXACT-PRO for capturing the initial symptoms of exacerbation and assessment of the recovery from exacerbation.
Chronic bronchitis, a type of COPD that is defined by productive cough for at least 3 months in at least two consecutive years, ${ }^{18}$ often can be a target of antibiotics for acute exacerbations. In this study, the EXACT scores were significantly higher in patients with chronic bronchitis. It suggests that COPD patients with chronic bronchitis may suffer from more severe symptoms than patients without chronic bronchitis.

We investigated NLR as a responsiveness marker during recovery from exacerbations after treatment. NLR is a good laboratory inflammatory marker, because it is cost-effective, rapid, easily available worldwide. ${ }^{19,20}$ NLR values were significantly higher in patients with COPD exacerbations than in stable COPD patients and controls ${ }^{19,20}$ and were significantly decreased during the convalescent period in patients with COPD exacerbations. ${ }^{20}$ In this study, NLR decreased in responders and increased in non-responders. Taylan et al ${ }^{19}$ suggested that NLR can be a marker for the early detection of exacerbations. We consider that NLR changes can be a sensitive laboratory measure for the assessment of recovery from exacerbations.

This study has several limitations. First, we did not enroll patients with unreported exacerbations; thus, the impact of EXACT on unreported exacerbations was not investigated. Many exacerbations are not reported ${ }^{2}$ and many patients suffer from worsening of unreported exacerbations and are treated late. Second, unfortunately, we did not collect baseline EXACT scores at the stable state before exacerbations, although we collected the EXACT scores on day 36 after recovery. Thus, we could not evaluate the relationship among the EXACT scores at baseline at the stable state, during exacerbation, and after recovery. Third, this study was performed in patients with mild-to-moderate exacerbations at outpatient clinic, not in admitted patients with severe exacerbations. Fourth, EXACT was designed and tested as an eDiary on a handheld device, and there are no available data on the performance characteristics of EXACT in the form of a paper-pen diary; ${ }^{3}$ however, we used a paper-pen diary booklet. Limitations of paper-pen diaries are the inability to determine subjects' compliance with daily data entry, to track subjects' entries or compliance in real time, and to prevent skipping items or marking $>1$ response for the same question. ${ }^{3}$

Nonetheless, to the best our knowledge, this study is the first report to measure EXACT scores from exacerbation onset to recovery after treatment with antibiotics in COPD patients in a Phase III randomized controlled clinical trial. Furthermore, this is the first report to use the Korean version of EXACT-PRO to assess treatment responsiveness following COPD exacerbations in Korea. 


\section{Conclusion}

This study shows that the EXACT scores had good internal consistency; decreased significantly after therapy in responders; were significantly correlated with the CAT score; were significantly higher in patients with chronic bronchitis; and in addition, we found that NLR decreased during recovery from exacerbations. Furthermore, we showed that the Korean version EXACT-PRO is valid, reliable, and sensitive to changes during recovery from exacerbations. We suggest the use of EXACT-PRO in patients with COPD exacerbations to assess the efficacy of acute treatment.

\section{Ethics approval and informed consent}

This study was approved by all Institutional Review Boards (IRBs) of participating hospitals, including Chungnam National University Hospital IRB. This study was registered with ClinicalTrials.gov number NCT01658020 and Clinical Research Information Service registry number KCT0000532. In Korea, there is no main approval IRB system in the case of multicenter study. Each participating hospital needs its own IRB approval. Therefore, this study protocol was approved by the individual IRBs of each hospital enrolling patients. All IRB names are listed as follows: Chungnam National University Hospital IRB, Chonbuk National University Hospital IRB, Chosun University Hospital IRB, CHA Bundang Medical Center IRB, Chungbuk National University Hospital IRB, Kangdong Sacred Heart Hospital IRB, Hanyang University Guri Hospital IRB, Asan Medical Center IRB, Gachon University Gil Medical Center IRB, Catholic University of Korea, Seoul ST Mary's Hospital IRB, Konyang University Hospital IRB, Gangneung Asan Hospital IRB, Kangwon National University Hospital IRB, Gyeongsang National University Hospital IRB, Kyunghee University Hospital IRB, Korea University Medical Center IRB, Dongguk University Gyeongju Hospital IRB, and Soonchunhyang University Hospital IRB. CNU-IRB file number is 2012-07-013. All patients provided written informed consent. All procedures performed in this study involving human participants were in accordance with the ethical standards of the institutional and/or national research committee and with the 1964 Declaration of Helsinki and its later amendments or comparable ethical standards.

\section{Data sharing statement}

We shall make data available to the scientific community with as few restrictions as feasible, while retaining exclusive use until the publication of major outputs. All available data can be obtained by contacting the corresponding author. Proposals should be directed to sungsoojung09@gmail.com.
To gain access, data requestors will need to sign a data access agreement. Individual participant data that underlie the results reported in this article, after deidentification (text, tables, figures, and appendices), will be made available. Other study-related documents (study protocol, statistical analysis plan, and analytic code) will be made available. Data sharing will be held beginning 9 months and ending 36 months following article publication.

\section{Disclosure}

The authors report no conflicts of interest in this work.

\section{References}

1. Vogelmeier CF, Criner GJ, Martinez FJ, et al. Global strategy for the diagnosis, management, and prevention of chronic obstructive lung disease 2017 report. Gold executive summary. Am J Respir Crit Care Med. 2017;195(5):557-582.

2. Langsetmo L, Platt RW, Ernst P, Bourbeau J. Underreporting exacerbation of chronic obstructive pulmonary disease in a longitudinal cohort. Am J Respir Crit Care Med. 2008;177(4):396-401.

3. EXACT-PRO Initiative; October 2014. The exacerbations of chronic pulmonary disease tool (exact) patient-reported outcome (pro) user manual (version 7.0). Available from: http://www.ema.europa.eu/docs/ en_GB/document_library/Other/2015/04/WC500185444.pdf. Accessed February 25, 2017.

4. Leidy NK, Wilcox TK, Jones PW, et al; EXACT-PRO Study Group. Development of the exacerbations of chronic obstructive pulmonary disease tool (exact): a patient-reported outcome (pro) measure. Value in Health. 2010;13(8):965-975.

5. Mackay AJ, Donaldson GC, Patel AR, Singh R, Kowlessar B, Wedzicha JA. Detection and severity grading of COPD exacerbations using the exacerbations of chronic pulmonary disease tool (exact). Eur Respir J. 2014;43(3):735-744.

6. Leidy NK, Murray LT, Jones P, Sethi S. Performance of the exacerbations of chronic pulmonary disease tool patient-reported outcome measure in three clinical trials of chronic obstructive pulmonary disease. Ann Am Thorac Soc. 2014;11(3):316-325.

7. Leidy NK, Wilcox TK, Jones PW, et al; EXACT-PRO Study Group. Standardizing measurement of chronic obstructive pulmonary disease exacerbations. reliability and validity of a patient-reported diary. Am J Respir Crit Care Med. 2011;183(3):323-329.

8. Rhee CK, Chang JH, Choi EG, et al. Zabofloxacin versus moxifloxacin in patients with COPD exacerbation: a multicenter, double-blind, double-dummy, randomized, controlled, phase III, non-inferiority trial. Int J Chron Obstruct Pulmon Dis. 2015;10:2265-2275.

9. Burge S, Wedzicha JA. COPD exacerbations: definitions and classifications. Eur Respir J Supplement. 2003;41:46s-53s.

10. Jones PW, Lamarca R, Chuecos F, et al. Characterisation and impact of reported and unreported exacerbations: results from ATTAIN. Eur Respir J. 2014;44(5):1156-1165.

11. Jung SS, Ys K, Choi HS. Usefulness of the exacerbations of chronic obstructive pulmonary disease tool-patient-report outcome (exact-Pro) measurement to assess exacerbations of COPD in Korea [abstract]. Am J Respir Crit Care Med. 2015:A2828.

12. Jones PW, Chen WH, Wilcox TK, Sethi S, Leidy NK; EXACT-PRO Study Group. Characterizing and quantifying the symptomatic features of COPD exacerbations. Chest. 2011;139(6):1388-1394.

13. Halpin DM, Laing-Morton T, Spedding S, et al. A randomised controlled trial of the effect of automated interactive calling combined with a health risk forecast on frequency and severity of exacerbations of COPD assessed clinically and using exact pro. Prim Care Respir J. 2011;20(3):324.

14. Nunnally JC, Bernstein IH. The Assessment of Reliability. Psychometric Theory. New York: McGraw-Hill; 1994. 
15. Jones PW, Harding G, Berry P, Wiklund I, Chen WH, Kline Leidy N. Development and first validation of the COPD assessment test. Eur Respir J. 2009;34(3):648-654.

16. Agustí A, Soler JJ, Molina J, et al. Is the cat questionnaire sensitive to changes in health status in patients with severe COPD exacerbations? COPD. 2012;9(5):492-498.

17. Tu YH, Zhang Y, Fei GH. Utility of the cat in the therapy assessment of COPD exacerbations in China. BMC Pulm Med. 2014;14:42.

18. Pauwels RA, Buist AS, Calverley PM, Jenkins CR, Hurd SS, Cr J. GOLD Scientific Committee. Global strategy for the diagnosis, management, and prevention of chronic obstructive pulmonary disease. NHLBI/WHO global initiative for chronic obstructive lung Disease (Gold) workshop summary. Am J Respir Crit Care Med. 2001;163(5): 1256-1276.
19. Taylan M, Demir M, Kaya H, et al. Alterations of The neutrophillymphocyte ratio during the period of stable and acute exacerbation of chronic obstructive pulmonary disease patients. Clin Respir J. 2017; 11(3):311-317.

20. Lee SJ, Lee HR, Lee TW, et al. Usefulness of neutrophil to lymphocyte ratio in patients with chronic obstructive pulmonary disease: a prospective observational study. Korean J Intern Med. 2016;31(5):891-898.

\section{Publish your work in this journal}

The International Journal of COPD is an international, peer-reviewed journal of therapeutics and pharmacology focusing on concise rapid reporting of clinical studies and reviews in COPD. Special focus is given to the pathophysiological processes underlying the disease, intervention programs, patient focused education, and self management protocols.

\section{Dovepress}

This journal is indexed on PubMed Central, MedLine and CAS. The manuscript management system is completely online and includes a very quick and fair peer-review system, which is all easy to use. Visit $\mathrm{http}: / / \mathrm{www}$.dovepress.com/testimonials.php to read real quotes from published authors.

\footnotetext{
Submit your manuscript here: http://www.dovepress.com/international-journal-of-chronic-obstructive-pulmonary-disease-journal
} 